\title{
ABOUT SOME PROPERTIES OF JACOBIAN FOR POLYNOMIAL MAPPINGS OF TWO COMPLEX VARIABLES
}

\author{
Bartosz Gawroński ${ }^{1}$, Julia Jankowska ${ }^{1}$, Rafat Moltzan ${ }^{1}$, Izabela Wtodarczyk ${ }^{1}$ \\ Grzegorz, Biernat ${ }^{2}$ \\ ${ }^{I}$ The third year students of Mathematics \\ ${ }^{2}$ Institute of Mathematics, Czestochowa University of Technology \\ Czestochowa, Poland \\ ${ }^{1}$ matematyka.studia@gmail.com
}

\begin{abstract}
In our article we consider jacobian $\operatorname{Jac}(f, h)$ of polynomial mapping $f=X^{k} Y^{k}+\ldots+$ $+f_{1}, h=X^{k-1} Y^{k-1}+\ldots+h_{1}$. We give conditions for coordinate $h$ in which constant jacobian $\operatorname{Jac}(f, h)=\operatorname{Jac}\left(f_{1}, h_{1}\right)$ vanishes.
\end{abstract}

Keywords: polynomial mappings, jacobian

\section{Introduction}

One of the most interesting issues of classical algebraic geometry is the question of polynomial mapping of two complex variables with constant jacobian zeros at infinity quantity [1]. In article [2] the authors showed that this kind of mapping has at most two zeros at infinity. In our article we give the conditions in which mapping in constant jacobian has one zero at infinity.

\section{Selected properties of jacobians}

To simplify the properties we assume that $f$ and $g$ are forms.

\section{Property 1}

We have following formulas:

1.1. Formula

$$
\operatorname{Jac}\left(X^{p} Y^{q}, X^{r} Y^{s}\right)=\left|\begin{array}{ll}
p & q \\
r & s
\end{array}\right| X^{p+r-1} Y^{q+s-1}
$$


1.2. Formula

$$
\begin{aligned}
& \operatorname{Jac}\left(X^{p} Y^{p} f, X^{q} Y^{q} g\right)= \\
& p X^{p+q-1} Y^{p+q-1} \operatorname{fJac}(X Y, g)- \\
& q X^{p+q-1} Y^{p+q-1} g \operatorname{Jac}(X Y, f)+ \\
& X^{p+q} Y^{p+q} \operatorname{Jac}(f, g)
\end{aligned}
$$

1.3. If $f$ and $g$ are $2 k$ - 1 -degree, then

$$
\operatorname{Jac}(X Y, f)=\operatorname{Jac}(X Y, g) \Leftrightarrow f=g
$$

1.4. If $f$ and $g$ are $2 k$-degree forms, then

$$
\operatorname{Jac}(X Y, f)=\operatorname{Jac}(X Y, g) \Leftrightarrow f=g+a_{k} X^{k} Y^{k}
$$

1.5. Formula

$$
\operatorname{Jac}\left(X Y, f^{k}\right)=k f^{k-1} \operatorname{Jac}(X Y, f)
$$

1.6. Formula

$$
\operatorname{Jac}(X Y, f \cdot g)=f J a c(X Y, g)+g J a c(X Y, f)
$$

1.7. Formula

$$
\begin{aligned}
& \operatorname{Jac}\left(X Y, g_{1}{ }^{k}\right)=0 \Leftrightarrow g_{1}=0, k \geq 1 \\
& k g_{1}{ }^{k-1} \operatorname{Jac}\left(X Y, g_{1}\right)=0 \Rightarrow g_{1}=0 \operatorname{or} J a c\left(X Y, g_{1}\right)=0 \Rightarrow g_{1}=0
\end{aligned}
$$

\section{The simplest examples}

\subsection{Example 1}

To study the simplest case, we must assume that polynomials $f=X^{2} Y^{2}+f_{3}+f_{2}+$ $+f_{1}$ and $h=X Y+h_{1}$ have constant jacobian $\operatorname{Jac}(f, h)=\operatorname{Jac}\left(f_{1}, h_{1}\right)$.

Then in sequence

1) $\operatorname{Jac}\left(X^{2} Y^{2}, h_{1}\right)+\operatorname{Jac}\left(f_{3}, X Y\right)=0$

therefore

$2 X Y J a c\left(X Y, h_{1}\right)=\operatorname{Jac}\left(X Y, f_{3}\right)$

so

$$
\operatorname{Jac}\left(X Y, 2 X Y h_{1}\right)=\operatorname{Jac}\left(X Y, f_{3}\right)
$$


and then

$f_{3}=2 X Y h_{1}$

2) $\operatorname{Jac}\left(f_{3}, h_{1}\right)+\operatorname{Jac}\left(f_{2}, X Y\right)=0$

therefore

$\operatorname{Jac}\left(2 X Y h_{1}, h_{1}\right)=\operatorname{Jac}\left(X Y, f_{2}\right)$

so

$\operatorname{Jac}\left(X Y, h_{1}^{2}\right)=\operatorname{Jac}\left(X Y, f_{2}\right)$

and then

$f_{2}=h_{1}^{2}+a_{1} X Y$

3) $\operatorname{Jac}\left(f_{2}, h_{1}\right)+\operatorname{Jac}\left(f_{1}, X Y\right)=0$

therefore

$\operatorname{Jac}\left(h_{1}^{2}+a_{1} X Y, h_{1}\right)=\operatorname{Jac}\left(X Y, f_{1}\right)$

so

$\operatorname{Jac}\left(X Y, a_{1} h_{1}\right)=\operatorname{Jac}\left(X Y, f_{1}\right)$

and then

$f_{1}=a_{1} h_{1}$

This means that $\operatorname{Jac}\left(f_{1}, h_{1}\right)=0$, the simplest polynomial having two zeros at infinity cannot have a constant nonzero jacobian (if it has a constant jacobian then the jacobian equals zero).

We may notice that in this elementary example

$f=h+a_{1} h$

$f=X^{2} Y^{2}+2 X Y h_{1}+\left(h_{1}^{2}+a_{1} X Y\right)+a_{1} h_{1}=\left(X Y+h_{1}\right)^{2}+a_{1}\left(X Y+h_{1}\right)$

This solution confirms the fact that jacobian $\operatorname{Jac}(f, h)=0$ (polynomials $f$ and $h$ are algebraically dependent in this trivial case).

\subsection{Example 2}

In the next example we may suppose that polynomials $f=X^{2} Y^{2}+f_{5}+f_{4}+f_{3}+$ $+f_{2}+f_{1}$ and $h=X^{2} Y^{2}+h_{3}+h_{2}+h_{1}$ have constant jacobian. For the reader it might be difficult so let us try to move the pattern from example 1. In sequence

1) $\operatorname{Jac}\left(X^{3} Y^{3}, h_{3}\right)+\operatorname{Jac}\left(f_{5}, X^{2} Y^{2}\right)=0$

therefore

$3 X^{2} Y^{2} \operatorname{Jac}\left(X Y, h_{3}\right)=2 X Y J a c\left(X Y, f_{5}\right)$

so

$3 X Y J a c\left(X Y, h_{3}\right)=2 \operatorname{Jac}\left(X Y, f_{5}\right)$

and then

$f_{5}=\frac{3}{2} X Y h_{3}$ 
2) $\operatorname{Jac}\left(X^{3} Y^{3}, h_{2}\right)+\operatorname{Jac}\left(f_{5}, h_{3}\right)+\operatorname{Jac}\left(f_{4}, X^{2} Y^{2}\right)=0$

therefore

$3 X^{2} Y^{2} \operatorname{Jac}\left(X Y, h_{2}\right)+\operatorname{Jac}\left(\frac{3}{2} X Y h_{3}, h_{3}\right)=2 X Y J a c\left(X Y, f_{4}\right)$

so

$\operatorname{Jac}\left(X Y, 3 X^{2} Y^{2} h_{2}\right)+\operatorname{Jac}\left(X Y, \frac{3}{4} h_{3}{ }^{2}\right)=\operatorname{Jac}\left(X Y, 2 X Y f_{4}\right)$

and then

$2 X Y f_{4}=3 X^{2} Y^{2} h_{2}+\frac{3}{4} h_{3}^{2}+a_{3} X^{3} Y^{3}$

it means that $X Y$ divides $h_{3}^{2}$, so it divides also $h_{3}$ so

$h_{3}=X Y h_{3 / 1}$

therefore

$2 X Y f_{4}=3 X^{2} Y^{2} h_{2}+\frac{3}{4} X^{2} Y^{2} h_{3 / 1}{ }^{2}+a_{3} X^{3} Y^{3}$

and then

$f_{4}=\frac{3}{2} X Y h_{2}+\frac{3}{8} X Y h_{3 / 1}^{2}+\frac{1}{2} a_{3} X^{2} Y^{2}$

3) $\operatorname{Jac}\left(X^{3} Y^{3}, h_{1}\right)+\underbrace{\operatorname{Jac}\left(f_{5}, h_{2}\right)}+\underbrace{\operatorname{Jac}\left(f_{4}, h_{3}\right.})+\operatorname{Jac}\left(f_{3}, X^{2} Y^{2}\right)=0$

(1) $=$

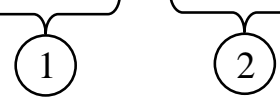

$\operatorname{Jac}\left(f_{5}, h_{2}\right)=\operatorname{Jac}\left(\frac{3}{2} X^{2} Y^{2} h_{3 / 1}, h_{2}\right)=3 X Y h_{3 / 1} \operatorname{Jac}\left(X Y, h_{2}\right)+\frac{3}{2} X^{2} Y^{2} J a c\left(h_{3 / 1}, h_{2}\right)$

(2) $=$

$\operatorname{Jac}\left(f_{4}, h_{3}\right)=\operatorname{Jac}\left(\frac{3}{2} X Y h_{2}+\frac{3}{8} X Y h_{3 / 1}{ }^{2}+\frac{1}{2} a_{3} X^{2} Y^{2}, X Y h_{3 / 1}\right)=$

$=\frac{3}{2} \operatorname{Jac}\left(X Y h_{2}, X Y h_{3 / 1}\right)+\frac{3}{8} \operatorname{Jac}\left(X Y h_{3 / 1}{ }^{2}, X Y h_{3 / 1}\right)+\frac{1}{2} a_{3} J a c\left(X^{2} Y^{2}, X Y h_{3 / 1}\right)=$

$=\frac{3}{2} X Y h_{2} \operatorname{Jac}\left(X Y, h_{3 / 1}\right)-\frac{3}{2} X Y h_{3 / 1} \operatorname{Jac}\left(X Y, h_{2}\right)+\frac{3}{2} X^{2} Y^{2} J a c\left(h_{2}, h_{3 / 1}\right)-$

$-\frac{3}{8} X Y h_{3 / 1}^{2} \operatorname{Jac}\left(X Y, h_{3 / 1}\right)+a_{3} X Y J a c\left(h_{2}, h_{3 / 1}\right)$

therefore

$3 X^{2} Y^{2} \operatorname{Jac}\left(X Y, h_{1}\right)+\frac{3}{2} X Y h_{3 / 1} \operatorname{Jac}\left(X Y, h_{2}\right)+\frac{3}{2} X Y h_{2} \operatorname{Jac}\left(X Y, h_{3 / 1}\right)-$

$-\frac{3}{8} X Y h_{3 / 1}{ }^{2} \operatorname{Jac}\left(X Y, h_{3 / 1}\right)+a_{3} X^{2} Y^{2} \operatorname{Jac}\left(X Y, h_{3 / 1}\right)=2 X Y J a c\left(X Y, f_{3}\right)$ 
So

$\operatorname{Jac}\left(X Y, 3 X Y h_{1}\right)+\operatorname{Jac}\left(X Y, \frac{3}{2} h_{3 / 1} h_{2}\right)-\frac{1}{8} \operatorname{Jac}\left(X Y, h_{3 / 1}{ }^{3}\right)+\operatorname{Jac}\left(X Y, a_{3} X Y h_{3 / 1}\right)=$

$=\operatorname{Jac}\left(X Y, 2 f_{3}\right)$

and then

$f_{3}=\frac{3}{2} X Y h_{1}+\frac{3}{4} h_{3 / 1} h_{2}-\frac{1}{16} h_{3 / 1}^{3}+\frac{1}{2} a_{3} X Y h_{3 / 1}$

4) $\operatorname{Jac}\left(f_{5}, h_{1}\right)+\operatorname{Jac}\left(f_{4}, h_{2}\right)+\operatorname{Jac}\left(f_{3}, h_{3}\right)+\operatorname{Jac}\left(f_{2}, X^{2} Y^{2}\right)=0$

By performing tedious calculations we obtain

$f_{2}=\frac{3}{4} h_{3 / 1} h_{1}+\frac{1}{2} a_{3} h_{2}+\frac{1}{2} a_{2} X Y+\frac{3}{8} b_{1}^{2} X Y$

while

$h_{2}=\frac{1}{4} h_{3 / 1}^{2}+b_{1} X Y$

5) $\operatorname{Jac}\left(f_{4}, h_{1}\right)+\operatorname{Jac}\left(f_{3}, h_{2}\right)+\operatorname{Jac}\left(f_{2}, h_{3}\right)+\operatorname{Jac}\left(f_{1}, X^{2} Y^{2}\right)=0$

By performing tedious calculations we obtain $f_{1}=\alpha_{1} h_{1}+\beta_{1} h_{3 / 1}$

6) $\operatorname{Jac}\left(f_{3}, h_{1}\right)+\operatorname{Jac}\left(f_{2}, h_{2}\right)+\operatorname{Jac}\left(f_{1}, h_{3}\right)=0$

By performing tedious calculations we obtain

$h_{1}=\frac{1}{2} B_{1} h_{3 / 1}$

Therefore we have received

$h_{1}=\frac{1}{2} B_{1} h_{3 / 1}, h_{2}=\frac{1}{4} h_{3 / 1}{ }^{2}+B_{1} X Y$ and $h_{3}=X Y h_{3 / 1}$, which means, that

$h=X^{2} Y^{2}+X Y h_{3 / 1}+\frac{1}{4} h_{3 / 1}^{2}+B_{1} X Y+\frac{1}{2} B_{1} h_{3 / 1}=\left(X Y+\frac{1}{2} h_{3 / 1}\right)^{2}+B_{1}\left(X Y+\frac{1}{2} h_{3 / 1}\right)$

Also consequently

$f=\left(X^{3} Y^{3}+\frac{1}{2} h_{3 / 1}\right)^{3}+A_{1}\left(X Y+\frac{1}{2} h_{3 / 1}\right)^{2}+A_{2}\left(X Y+\frac{1}{2} h_{3 / 1}\right)$

By selecting right parameters $A_{1}=\frac{1}{2} a_{3}$ and

$A_{2}=\frac{1}{2} a_{2}+\frac{3}{2} b_{1} X Y+\frac{1}{2} a_{3} b_{1}+\frac{3}{8} b_{1}^{2}+\frac{3}{4} b_{1} h_{3 / 1}$ this means that

$\operatorname{Jac}\left(f_{1}, h_{1}\right)=\operatorname{Jac}\left(\frac{1}{2} A_{1} h_{3 / 1}, \frac{1}{2} B_{1} h_{3 / 1}\right)=0$

These examples show the general method. 


\section{Proposition}

Let $f=X^{k} Y^{k}+f_{2 k-1}+\ldots+f_{1}$, and $h=X^{k-1} Y^{k-1}+h_{2 k-3}+\ldots+h_{1}, k \geq 2$

If $\operatorname{Jac}(f, h)=\operatorname{Jac}\left(f_{1}, h_{1}\right)$, then $\operatorname{Jac}\left(f_{1}, h_{1}\right)=0$

The idea of the proof. The particular parts of the proof lead to the following forms of polynomials $f$ and $h$

$$
f=\left(X Y+\frac{1}{k-1} h_{2 k-3 / 1}\right)^{k}+A_{1}\left(X Y+\frac{1}{k-1} h_{2 k-3 / 1}\right)^{k-1}+\ldots+A_{k-1}\left(X Y+\frac{1}{k-1} h_{2 k-3 / 1}\right)
$$

And

$$
h=\left(X Y+\frac{1}{k-1} h_{2 k-3 / 1}\right)^{k-1}+B_{1}\left(X Y+\frac{1}{k-1} h_{2 k-3 / 1}\right)^{k-2}+\ldots+B_{k-2}\left(X Y+\frac{1}{k-1} h_{2 k-3 / 1}\right)
$$

While

$$
h_{2 k-3}=X^{k-2} Y^{k-2} h_{2 k-3 / 1}
$$

This means that polynomials $f$ and $h$ are algebraically dependent and it confirms that

$$
\operatorname{Jac}(f, h)=0
$$

\section{Conclusions}

We have received the whole set of polynomials formulas having two zeros at infinity in which the constant jacobian must vanish. We suppose that this is true for every polynomial mapping of two zeros at infinity. This note will be the aim of our next article.

\section{References}

[1] McKay J.H., Sui-Sheng Wang S., A note on the Jacobian condition and two points at infinity, Proceedings of the American Mathematical Society 1991, Jan., 111, 1, 35-43.

[2] Charzyński Z., Chądzyński J., Skibiński P., A contribution to Keller's Jacobian conjecture, Seminar on deformations (Proceedings, Łódź-Warsaw 1982/84, Lecture Notes in Mathematics 1165), Springer-Verlag, Berlin, Heidelberg, New York, and Tokyo 1985, 36-51. 